\title{
Estudo de orientação de partículas em aglomerados gerados por modelos bidimensionais
}

\section{(A study of particle orientation in agglomerates simulated by two-dimensional models)}

\author{
Vanderley de Vasconcelos, Lúcio Carlos Martins Pinto \\ Centro de Desenvolvimento da Tecnologia Nuclear - CDTN/CNEN \\ Caixa Postal 941, CEP 30.123-970, Belo Horizonte, MG \\ vasconv@urano.cdtn.br \\ pintolc@urano.cdtn.br \\ Wander L. Vasconcelos \\ Universidade Federal de Minas Gerais - Dep. Eng. Metalúrgica e Materiais \\ Rua Espírito Santos, 35, CEP 30.160-030, Belo Horizonte, MG \\ wlv@urano.cdtn.br
}

\begin{abstract}
Resumo
Na formação de aglomerados, partículas pequenas tendem a se agregar, reduzindo a energia livre total do sistema. Mesmo quando as partículas primárias têm forma esférica e tamanhos uniformes, dependendo do tipo do processamento e das forças envolvidas, os aglomerados podem apresentar orientações preferenciais de partículas. Este trabalho utiliza técnica de processamento digital de imagens para a obtenção das distribuições de orientação de partículas em aglomerados bidimensionais simulados. As simulações foram realizadas em um ambiente de computação gráfica com recursos de modelagem de sólidos. A escolha de modelos bidimensionais para representar casos tridimensionais é um artifício muito empregado para reduzir os altos tempos de processamento computacional envolvidos no estudo de processos complexos. Apesar de simples, em príncipio, muitas conclusões importante podem ser extraídas dos modelos bidimensionais e muitos de seus resultados aplicados diretamente aos casos reais.
\end{abstract}

\section{INTRODUÇÃO}

As propriedades de um material policristalino, em particular aquelas relacionadas à anisotropia mecânica, dependem fortemente da microestrutura [1]. Quando ocorre a formação de aglomerados, a densidade de empacotamento das partículas varia de região para região da amostra. No entanto, a distribuição do pó é essencial para determinar os mecanismos de densificação, especialmente nos casos onde a tensão desempenha um papel importante, tal como o caso da sinterização a alta pressão isostática (HIP).

Características de fluxo de partículas muito finas são também afetadas pela aglomeração de partículas. Com a finalidade de

\begin{abstract}
In the formation of agglomerates, small particles tend to aggregate in order to reduce the total free energy of the system. Even when the individual particles can be treated as spheres of uniform size, depending on the process and involved forces the agglomerates can show some kind of particle orientation. This paper uses digital image processing techniques for obtaining particle orientation distribution of two-dimensional simulated agglomerates. These simulations were carried out using a computer graphics environment with solid modeling extension. The use of two-dimensional models instead of threedimensional ones is an alternative to reduce the long computacional times involved in the analysis of complex real systems. Despite being simple, in principle, very important information can be obtained from twodimensional systems and directly applied to real threedimensional cases.
\end{abstract}

criar grãos para fluírem livremente, essas partículas são comumente aglomeradas em formas e tamanhos reprodutíveis através de alguma técnica de granulação adequada [2]. Apesar de grande incidência de sistemas nos quais alguma forma de aglomeração ocorre, os mecanismos que governam a sua formação, os quais dependem das forças entre as partículas, aglomerados e paredes dos recipientes, são, em geral, pouco conhecidos [3]. Isto é devido, em parte, à ausência de instrumentos laboratoriais para caracterizá-los adequadamente e também à alta capacidade computacional exigida para levar em conta as interações de milhões de partículas e aglomerados de propriedades muitas vezes heterogêneas.

Mesmo quando as partículas primárias têm forma esférica e 
tamanhos uniformes, dependendo do tipo do processamento e das forças envolvidas, os aglomerados podem apresentar orientações preferenciais de partículas. Os métodos convencionais utilizados para medir a orientação de grãos em materiais policristalinos, como a rosa dos interceptos [4], por exemplo, quando aplicados diretamente a estruturas do tipo dos aglomerados, não identificam quaisquer direções. Esses aglomerados são, muitas vezes, descritos como fractais e exibem dimensões fractais inferiores à sua dimensão euclidiana [3]. O propósito deste trabalho é duplo: desenvolver um modelo simples de simulação de aglomeração bidimensional de partículas de tal modo que a estrutura obtida possa ser caracterizada integralmente; e implementar um novo método de avaliação da orientação da estrutura formada pelas partículas no aglomerado.

\section{SIMULAÇÃO DA AGLOMERAÇÃO DE PARTÍCULAS}

Grupos de partículas fracamente ligadas podem se comportar como pseudopartículas maiores chamadas aglomerados. No caso de pós cerâmicos extremamente finos, a densidade de empacotamento é geralmente muito baixa. Uma das possíveis razões para isto é que as unidades de empacotamento básicas são aglomerados duros (agregados de baixa densidade formados durante a síntese do pó).

Esses agregados crescem à custa do acúmulo, em posições aleatórias, de pequenas partículas em movimento browniano em torno de um núcleo original. Dados da literatura indicam que tais agregados são auto-similares com o aumento de seus tamanhos, comportando-se, portanto, como fractais.

Neste trabalho foi utilizado um algoritmo bastante simples para a simulação geométrica de aglomerado de partículas: esferas são atraídas de posições aleatórios, em torno de uma esfera posicionada no centro de um recipiente bidimensional de dimensões pré-definidas (aqui denominado de centro de nucleação), até que atingem outra esfera [5].

Este procedimento simula a situação onde existem forças atrativas entre as partículas (forças de van der Waals ou eletrostáticas, por exemplo), as quais são responsáveis pela interrupção da movimentação de cada partícula. A Fig. 1 apresenta um aglomerado bidimensional simulado com esse algoritmo. As simulações foram realizadas em um ambiente de computação gráfica com recursos

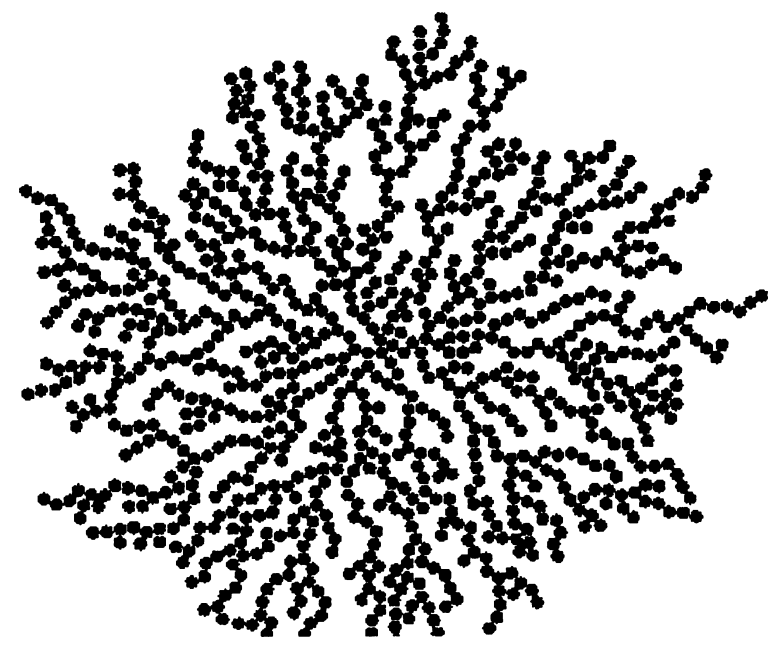

Figura 1: Aglomerado bidimensional simulado em um ambiente de computação gráfica e modelagem de sólidos.

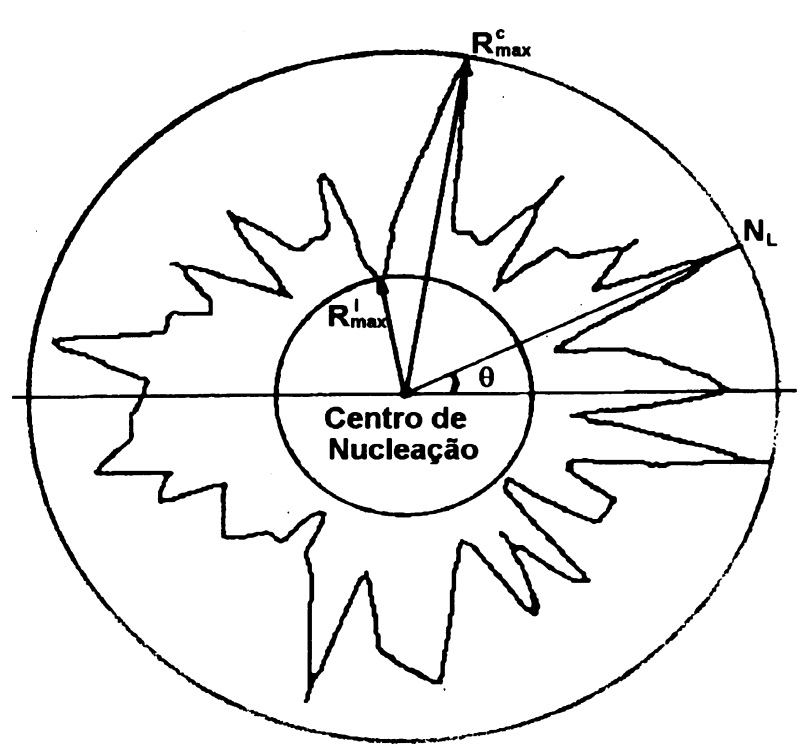

Figura 2: Orientação radial do aglomerado bidimensional simulado, obtida tomando como referência o centro de nucleação do aglomerado.

de modelagem de sólidos. A utilização deste ambiente, além de permitir o posicionamento e visualização de objetos empregando funções já existentes, possibilita a implementação de algoritmos específicos de movimentação e interação entre partículas através da programação em linguagens de alto nível, como LISP ou C.

\section{ESTUDO DE ORIENTAÇÃO NO AGLOMERADO}

Podem ser obtidas distribuições de orientações de linhas ou superfícies, e.g., orientações de contornos de grãos, arestas de grãos, contornos de fases, etc. Deve-se ressaltar que orientações de elementos microestruturais podem ser diferentes das orientações preferenciais dos cristais. Embora orientação preferencial, no sentido cristalino possa ser entendida como uma propriedade global, até agora não existe nenhuma maneira proposta de expressar orientações preferenciais através de algum parâmetro específico [1].

Uma forma de visualizar a distribuição de orientação é através da rosa dos interceptos [4]. Para sua construção em um sistema de linhas com um eixo de orientação, basta traçar no microscópio uma série de secantes paralelas, formando um ângulo definido com o eixo de orientação. É então determinado o número de interseções por unidade de comprimento, $\mathrm{N}_{\mathrm{L}}$, para este ângulo em particular. $\mathrm{O}$ processo é repetido por todas as direções do plano. É traçado, então, em coordenadas polares, o gráfico $\theta \mathrm{x} \mathrm{N}_{\mathrm{L}}$ e conectados os raios por curvas suaves, obtendo a conhecida rosa dos interceptos. Para um sistema isométrico (sem orientação) a rosa dos interceptos seria, de acordo com o procedimento descrito, uma circunferência com centro na origem do sistema polar. Aplicando-se esse método tradicional de medir a orientação de partículas ao aglomerado simulado, poder-se-ia concluir que neste caso não haveria nenhuma orientação preferencial. Torna-se necessária outra abordagem no sentido de levar em conta orientações radiais como as encontradas no aglomerado simulado. Neste trabalho sugere-se um modo alternativo de se medir a orientação, tomando-se como referência para o sistema de coordenadas polares o centro de nucleação. O método sugerido inclui a determinação do número de interceptos por unidade de comprimento de uma semi-reta com sua origem no centro de nucleação do aglomerado, em um numero $\mathrm{n}$ de direções. 
Assim, como incrementos de $2 \mu / \mathrm{n}$ radianos, são obtidos os valors $\mathrm{N}_{\mathrm{L}}$ para cada direção e traçado o gráfico de $\theta \times \mathrm{N}_{\mathrm{L}}$ em coordenadas polares. A Fig. 2 mostra o gráfico obtido para o aglomerado simulado da Fig. 1, para $\mathrm{n}=64$, após a conecção dos raios por uma envoltória. Este método foi implementado no analisador de imagens microestruturais Quantikov [6]. Através da análise do gráfico mostrado na Fig. 2 pode-se notar que o aglomerado simulado apresenta um grande número de eixos de orientação, caracterizados pelas direções com maiores raios. Pode-se definir um grau de orientação para cada direção $\theta, \omega_{\theta}$, como

$$
\omega_{\theta}=\frac{\mathrm{R}_{\theta}}{\mathrm{R}_{\text {max }}^{i}},
$$

onde $\mathrm{R}_{\theta}$ é o valor do raio $\left(\mathrm{N}_{\mathrm{L}}\right)$ para o ângulo e $\mathrm{R}_{\text {max }}^{\mathrm{i}}$ é o raio do maior círculo no diagrama com centro na origem do sistema polar.

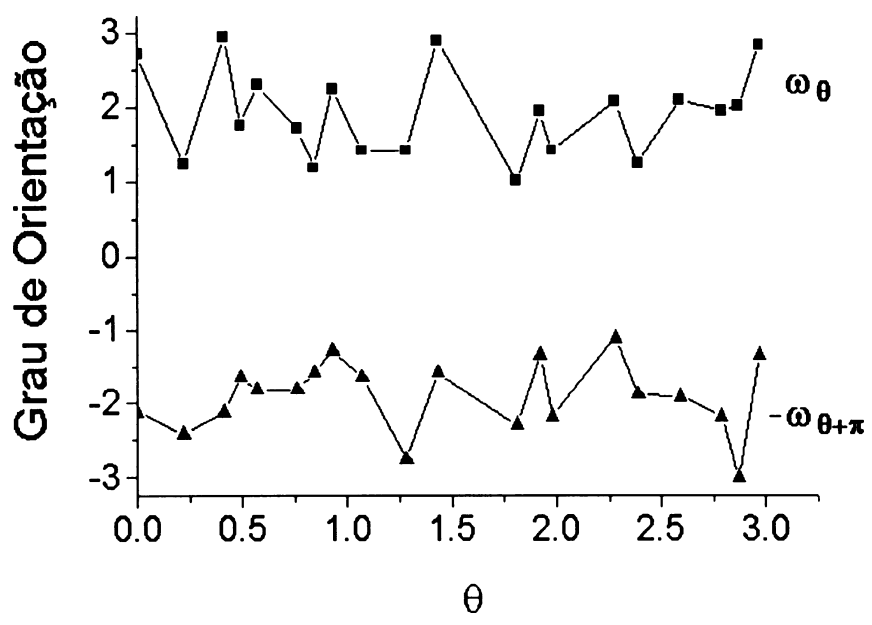

Figura 3: Variação do grau de orientação $\left(\omega_{\theta} e-\omega_{\theta+\pi}\right)$ para a orientação radial $\theta$, em radianos, para o aglomerado simulado.

Por esta definição, os valores de $\omega_{\theta}$ irão variar de $1\left(\mathrm{R}_{\theta}=\mathrm{R}_{\text {max }}^{\mathrm{i}}\right)$ até um valor máximo $\omega_{\theta}{ }^{\max }$, dado por

$$
\omega_{\theta}^{\max }=\frac{\mathrm{R}_{\text {max }}^{c}}{\mathrm{R}_{\text {max }}^{i}},
$$

onde $\mathrm{R}_{\text {max }}^{\mathrm{i}}$ é o maior círculo circunscrito no diagrama com centro na origem polar. Plotando em um mesmo gráfico em coordenadas cartezianas curvas independentes para $\omega_{\theta}$ e $\omega_{\theta+\pi}$ em função do angulo $\theta$, no intervalo $0 \leq \theta \leq \mu$ (Fig. 3 , além das orientações radiais, poderão ser identificadas regiões com um único eixo de orientação, bastando para isto avaliar a área entre duas curvas. Regiões com áreas bem acima das demais poderiam caracterizar regiões com um único eixo de orientação.

\section{CARACTERÍSTICAS FRACTAIS DO AGLOMERADO}

As características fractais do aglomerado bidimencional simulado, após algumas operações morfológicas de fechamento e afinamento utilizando o "software" Quantikov [6], foram analizadas utilizando, via internet, o "software" FRACTOP V0.1 [7]. Este

\section{FracTop V0.1}

Analysis - Report
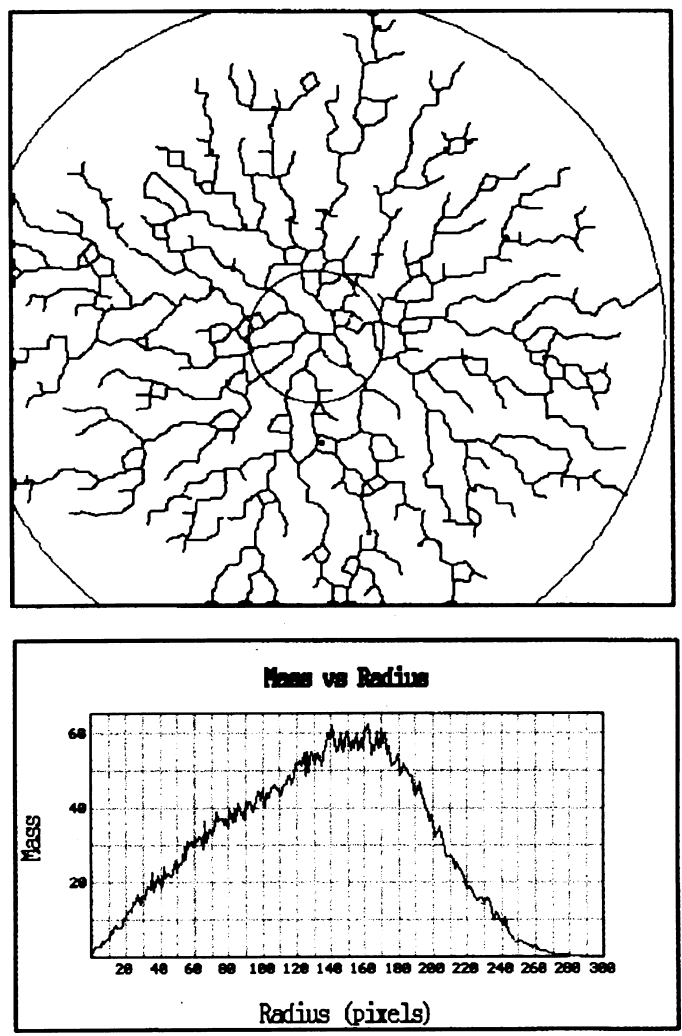

Figura 4: Variação da massa com o raio do aglomerado bidimensional simulado, ambos medidos em termos de número de pixels.
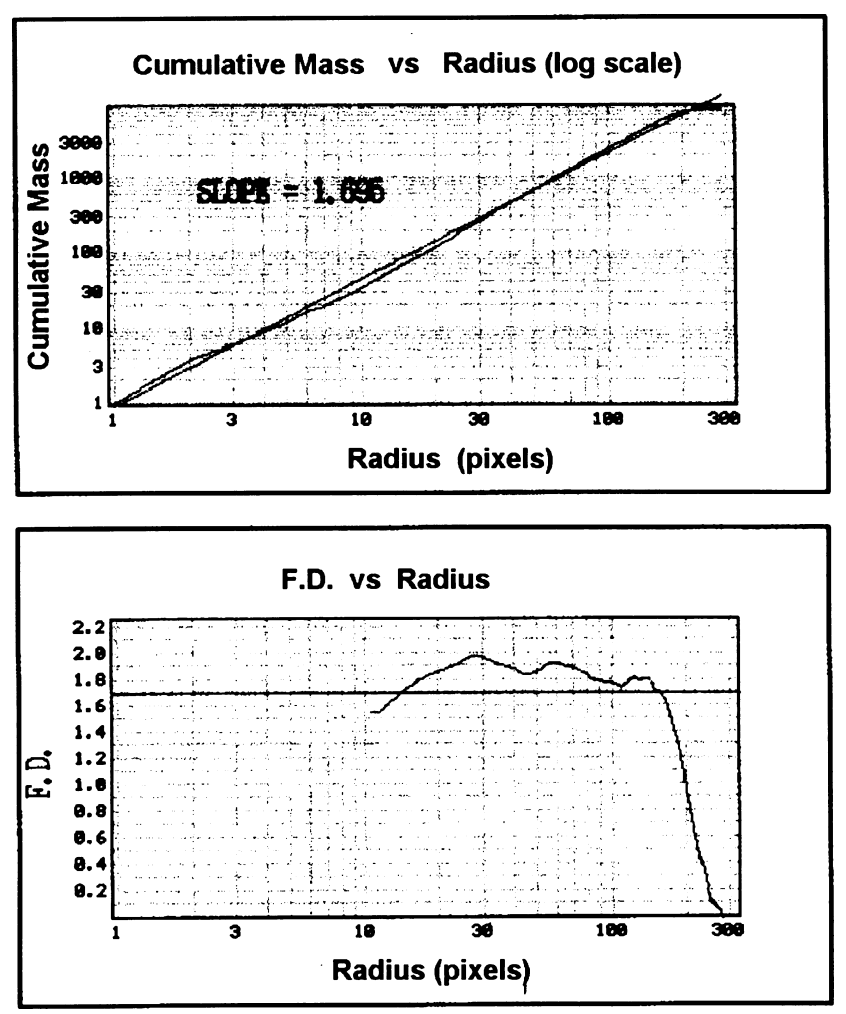

Figura 5: Variação da massa acumulada do aglomerado versus seu raio, utilizada para estimar a dimensão fractal (F. D.). 
"software" utiliza para a investigação da dimensão fractal um método baseado no acúmulo de massa. A Fig. 4 mostra a variação da massa com o raio do aglomerado, ambos medidos em termos de número de pixels. Nota-se que a massa do aglomerado cresce até um raio de aproximadamente 160 pixels, quando começa a reduzir, indo em torno de 280 pixels, quando o raio máximo do aglomerado é alcançado. Um gráfico logarítmico da variação da massa acumulada do aglomerado versus seu raio é utilizado para estimular a dimensão fractal (F. D. - "Fractal Dimension"na Fig. 5). Pela inclinação da reta ajustada à curva, pode-se verificar que o aglomerado se comporta como um fractal com dimensão 1,695, até o raio de aproximadamente 160 pixels.

\section{CONCLUSÃo}

A aplicação do método convencional de obtenção de distribuições de orientação no aglomerado simulado no ambiente de computação gráfica e modelagem de sólidos não detectou quaisquer orientações preferencias. A aplicação do método sugerido neste trabalho e implementado no analizador de imagens microestruturais Quantikov identificou orientações radiais do aglomerado simulado, através do posicionamento do sistema de coordenadas polares no seu centro de nucleação. A utilização do grau de orientação definido serve, não só para obtenção das direções radial, mas também para identificar outros possíveis eixos de orientação. Este mesmo conceito aplicado a estruturas sem orientação ou orientadas segundo um ou mais eixos de orientação produz resultados semelhantes aos obtidos pelo método convencional de obter a rosa dos interceptos. A interpretação desses aglomerados segundo conceitos fractais levou à determinação de um valor de 1,695 para a dimensão fractal. São necessários estudos adicionais no sentido de se tentar associar as características fractais desses aglomerados aos padrões gráficos obtidos com o novo método.

\section{AGRADECIMENTOS}

Os autores agradecem o apoio financeiro da FINEP/PADCT e FAPEMIG.

\section{REFERÊNCIAS}

[1] J. Pospiech, K. Lücke, K. Sztwiertnia, Acta Metallurgica et Materialia 41, 1 (1993) 305.

[2] N. Özkan, B. J. Briscoe, Powder Technology 86 (1995) 201.

[3] S. J. R. Simons, Powder Technology 87 (1996) 29.

[4] R. T. DeHoff, F. N. Rhines, Quantitative Microscopy, New

York, McGraw-Hill Book Company, (1968).

[5] V. Vasconcelos, W. L. Vasconcelos, Anais do XXXIX Congresso Brasileiro de Cerâmica, Águas de Lindóia, S. P., 1995, vol.1, p. 53.

[6] L.C.M. Pinto, Quantikov- Um analisador microestrutural para o ambiente Windows ${ }^{\mathrm{TM}}$. Tese de Doutorado, Universidade de São Paulo-USP, IPEN, (1996).

[7] D. G. Green, Fractop V. 01 - Complexity on-line interactive services. Environmental and Information Science, Charles Sturt University, Australia, 1993. (http//life.csu.edu.au/fractop/).

(Rec. 14/04/97, Ac. 01/03/98) 